\title{
Simvastatin attenuates radiation-induced salivary gland dysfunction in mice
}

\author{
This article was published in the following Dove Press journal: \\ Drug Design, Development and Therapy \\ 13 July 2016 \\ Number of times this article has been viewed
}

\author{
Liping Xu* \\ Xi Yang* \\ Jiayan Chen \\ Xiaolin Ge \\ Qin Qin \\ Hongcheng Zhu \\ Chi Zhang \\ Xinchen Sun \\ Department of Radiation Oncology, \\ The First Affiliated Hospital of \\ Nanjing Medical University, Nanjing, \\ People's Republic of China \\ *These authors contributed equally \\ to this work
}

Objective: Statins are widely used lipid-lowering drugs, which have pleiotropic effects, such as anti-inflammation, and vascular protection. In our study, we investigated the radioprotective potential of simvastatin (SIM) in a murine model of radiation-induced salivary gland dysfunction.

Design: Ninety-six Institute of Cancer Research mice were randomly divided into four groups: solvent + sham irradiation (IR) (Group I), SIM + sham IR (Group II), IR + solvent (Group III), and IR + SIM (Group IV). SIM (10 mg/kg body weight, three times per week) was administered intraperitoneally 1 week prior to IR through to the end of the experiment. Saliva and submandibular gland tissues were obtained for biochemical, morphological (hematoxylin and eosin staining and Masson's trichrome), and Western blot analysis at 8 hours, 24 hours, and 4 weeks after head and neck IR.

Results: IR caused a significant reduction of salivary secretion and amylase activity but elevation of malondialdehyde. SIM remitted the reduction of saliva secretion and restored salivary amylase activity. The protective benefits of SIM may be attributed to scavenging malondialdehyde, remitting collagen deposition, and reducing and delaying the elevation of transforming growth factor $\beta 1$ expression induced by radiation.

Conclusion: SIM may be clinically useful to alleviate side effects of radiotherapy on salivary gland.

Keywords: simvastatin, radiation protection, submandibular gland, transforming growth factor- $\beta 1$, mice

\section{Introduction}

Radiotherapy plays an important role in the treatment of malignant tumors of the head and neck. Despite new improvements in radiotherapy technique and equipment, it is impossible to spare the salivary glands, and the life quality of these patients is severely compromised because of radiation-induced impairment of salivary gland function. Patients with radiation-induced salivary gland dysfunction suffer from dysphagia, impaired sense of taste, increased mucosal infections, and other discomforts. Of all these complications, salivary gland dysfunction associated with irreversible xerostomia is one of the most important complications that impairs the quality of life significantly. ${ }^{1}$ Xerostomia can occur early during radiation therapy; a rapid decrease of the salivary flow rate is observed during the first week of radiation therapy, after which there is a continuing gradual decrease to $<10 \%$ of the initial flow rate. ${ }^{2}$ Patients affected by xerostomia have a hypofunction of the salivary output, leading to functional oral disorders such as sore throat, altered taste, dental decay, changes in voice quality, and impaired chewing and swallowing function. ${ }^{3}$ However, current management approaches remain palliative and are generally considered to be unsatisfactory.
Correspondence: Xinchen Sun Department of Radiation Oncology, The First Affiliated Hospital of Nanjing Medical University, 300 Guangzhou Road, Nanjing 210029, People's Republic of China

$\mathrm{Tel} / \mathrm{fax}+8602568135700$

Email sunxinchen2012@163.com
Drug Design, Development and Therapy 2016:10 227I-2278

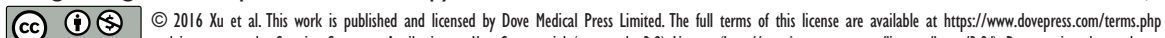
cc. hereby accept the Terms. Non-commercial uses of the work are permitted without any further permission from Dove Medical Press Limited, provided the work is properly attributed. For permission for commercial use of this work, please see paragraphs 4.2 and 5 of our Terms (https://www.dovepress.com/terms.php). 
The commonly used lipid-lowering compounds, statins, have pleiotropic effects, such as cholesterol-lowering, cardiovascular protection, and antioxidative properties. ${ }^{4-7} \mathrm{In}$ addition, it was reported that simvastatin (SIM) ameliorated radiation-induced enteritis and lung injury. ${ }^{8,9}$

The purpose of our study was to investigate the radioprotective effect of SIM on salivary gland and the possible mechanisms by which SIM exerted the radioprotective potential in mice.

\section{Materials and methods Animals}

Eight-week-old male Institute of Cancer Research (ICR) mice weighing $30 \pm 2 \mathrm{~g}$ purchased from Nanjing Medical University Laboratory Animal Center were used in this study and maintained in a specific pathogen-free environment. The mice were fed a standard pellet diet and allowed free access to water. During the study, mice were observed daily for general condition and activity levels. Any signs of abnormalities (depilation, weight loss, illness, or mortality) were recorded and investigated. All animal procedures were approved by the Animal Care and Use Committee of Nanjing Medical University.

\section{Treatment groups}

Using StataSE11.0 software to produce random numbers, a total of 96 ICR mice were randomly divided into four groups: solvent + sham irradiation (IR) (Group I), SIM (Sigma-Aldrich Co., St Louis, MO, USA) + sham IR (Group II), IR + solvent (Group III), and IR + SIM (Group IV). Mice were treated with $10 \mathrm{mg} / \mathrm{kg}$ SIM via intraperitoneal (IP) injection every 2 days beginning 1 week (Monday, Wednesday, Friday, and Sunday) before IR and continuing up to the end of the experiment. Particularly, SIM was given 30 minutes before IR on the first Sunday. Saliva and submandibular gland (SMG) tissues were obtained for biochemical, morphological, and Western blot analysis at 8 hours, 24 hours, and 4 weeks after localized IR of the head and neck.

\section{Radiation procedure}

Prior to IR, mice were anesthetized by an IP injection of $50 \mathrm{mg} / \mathrm{kg}$ sodium pentobarbital. Then, the mice were placed in a plastic tube holding them firmly in a supine position during IR. The head and neck regions were then locally irradiated with a single exposure to a 15 Gy electron beam from a medical linear accelerator (Elekta Precise, Stockholm, Sweden; $6 \mathrm{Mev}$, dose rate $=400 \mathrm{MU} / \mathrm{min}$, source skin distance $=100 \mathrm{~cm}$ ).
During the IR procedure, the rest of the body was shielded by a 12-mm-thick lead shield positioned on the chest and abdomen. The total radiation field used on the accelerator was $8 \times 20 \mathrm{~cm}$, allowing eight mice to be irradiated simultaneously.

\section{Saliva collection and salivary amylase activity analysis}

Saliva samples were collected for 15 minutes following stimulation with $2 \mathrm{mg} / \mathrm{kg}$ pilocarpine nitrate, which was administered in anesthetized mice by IP injection. Saliva was collected in preweighed ice-cooled microfuge tubes 8 hours, 24 hours, and 4 weeks after IR. The total volume of saliva secreted was estimated by weight as described previously. ${ }^{10}$ Saliva volume was then normalized to body weight to obtain a value for saliva flow. Salivary amylase (AMS) activity was measured by the iodinestarch colorimetric method using the AMS Assay Kit (Nanjing Jiancheng Bioengineering Institute, Nanjing, People's Republic of China) according to the manufacturer's instructions.

\section{Biochemical assays}

To ensure that SIM exerted its potency, blood samples were collected at 1 day and 28 days after IR, and then left at room temperature for 2 hours to allow coagulation. Subsequently, samples were centrifuged ( 10 minutes, $5,000 \times g$ ) to yield serum for the analysis of serum cholesterol using Total Cholesterol Reagent Kit (Dongou Diagnostics Co., Ltd, Zhejiang, People's Republic of China).

The content of malondialdehyde (MDA) was detected using the thiobarbituric acid method. Briefly, SMG samples were removed, cleared of blood, and immediately transferred to ice-cold containers containing $10 \mathrm{mM}$ phosphate-buffered saline. A known amount of tissue was homogenized using normal saline in a tissue homogenizer on ice. The homogenate was centrifuged at 3,000 rpm for 10 minutes at $4^{\circ} \mathrm{C}$, and the supernatant was used for the assay of MDA content using the MDA Assay Kit. The protein concentration was measured by the bicinchoninic acid method using protein quantification kit. All the indicators were measured at least three times from six samples according to the manufacturer's instructions (Jiancheng Bioengineering Institute, Nanjing, People's Republic of China).

\section{Histology}

The SMG tissues were fixed in 10\% neutral formalin and embedded in paraffin for histological sections. Tissue sections $(5 \mu \mathrm{m})$ were stained with hematoxylin and eosin (H\&E) and Masson's trichrome stains and then examined under a microscope with a camera. All tissue sections were 
examined for the characterization of histopathological changes by an experienced histologist who was unaware of the treatment conditions.

\section{Preparation of protein extracts and Western blot analysis}

For the preparation of total protein extracts, $\sim 60 \mathrm{mg} \mathrm{SMG} \mathrm{tissue}$ was homogenized in ice-cold cell lysis buffer for Western blot (Beyotime Institute of Biotechnology, Haimen, Jiangsu, People's Republic of China; $20 \mathrm{mM}$ Tris, $\mathrm{pH} 7.5,150 \mathrm{mM} \mathrm{NaCl}$, $1 \%$ Triton X-100, sodium pyrophosphate, $\beta$-glycerophosphate, $1 \mathrm{mM}$ ethylenediaminetetraacetic acid, $1 \mathrm{mM} \mathrm{Na}_{3} \mathrm{VO}_{4}, 1 \mathrm{mM}$ phenylmethylsulfonyl fluoride). Subsequently, the homogenate was centrifuged at $12,000 \times \mathrm{g}$ for 10 minutes at $4^{\circ} \mathrm{C}$, and the supernatant was collected for protein determination by bicinchoninic acid. Equal amounts of proteins were then separated by sodium dodecyl sulfate polyacrylamide gel electrophoresis before being transferred on to polyvinylidene fluoride membranes (EMD Millipore, Billerica, MA, USA). The membranes were blocked in a blocking buffer ( $5 \%$ nonfat milk, $1 \%$ Tween 20, in $20 \mathrm{mM}$ Tris-buffered saline, $\mathrm{pH}$ 7.6) for 1 hour at room temperature, followed by incubation with the transforming growth factor (TGF)- $\beta 1$ rabbit polyclonal immunoglobulin G (IgG) (Santa Cruz Biotechnology Inc., Dallas, TX, USA) in a blocking buffer overnight at $4^{\circ} \mathrm{C}$. This was followed by incubation with horseradish peroxidase-conjugated anti-rabbit secondary antibody (Bioworld Technology Co., Ltd, St Louis Park, MN, USA) for 1 hour, and then washing three times, and detection by ChemiDoc ${ }^{\mathrm{TM}} \mathrm{XRS}^{+}$system (Bio-Rad Laboratories Inc., Hercules, CA, USA) according to the manufacturer's instructions. The density measurement of each band was obtained using Image Lab software (Bio-Rad Laboratories Inc.). Values were expressed as fold change relative to control and normalized to $\beta$-actin, the loading control (Beyotime Institute of Biotechnology).

\section{Statistical analysis}

Statistical analysis was performed using SPSS 18.0 software (SPSS Inc., Chicago, IL, USA). All results were expressed as the mean \pm standard deviation. Analysis of variance was used to compare differences among each group by Dunnett's tests for multiple comparisons. A value of $P<0.05$ was considered statistically significant.

\section{Results}

There were no significant differences in water in take, food consumption, or weight gain among the four groups during the follow-up period after IR. No deaths occurred.

\section{SIM prevents radiation-induced salivary gland hypofunction}

To investigate salivary gland function, saliva was collected for 15 minutes after pilocarpine injection and assessed at intervals of 8 hours, 24 hours, and 4 weeks after IR (Figure 1A). At 8 hours, salivary flow in Group III was significantly reduced when compared to Group I (decreased by $19.07 \%, P<0.01)$, but there was no clear reduction in Group IV $(P=0.993)$ compared with Group I. At 24 hours and 4 weeks, both Group III and Group IV showed a significant reduction of salivary flow ( $41.6 \%$ vs $28.7 \%, 61 \%$ vs $54.5 \%$ ) when compared to Group I.

The salivary flow of Group III demonstrated a progressive and significant time-dependent decrease with time after IR, suggesting that the irradiated mice developed acute ( 8 hours, 24 hours) and subacute (30 days) hyposalivation compared with the normal mice (Figure 1A, $P<0.01$ ). In contrast, there was no significant difference in saliva flow between Group I and Group IV 8 hours after IR. Although the salivary flow in Group IV began to decline 24 hours after IR and continued to show hyposalivation at 4 weeks, the extent of reduction in Group IV was less pronounced than that of Group III at either of the two time intervals $(P<0.01, P>0.05)$.

For salivary AMS (Figure 1B), there were no significant differences between any of the groups at 8 hours and 24 hours after IR. But, at 4 weeks, the salivary AMS activity of Group III was significantly reduced when compared with Group I $(P<0.01)$. However, the salivary AMS activity of Group IV showed a significant increment compared with Group I at 4 weeks $(P<0.01)$.

\section{Effects of lowering serum cholesterol and scavenging SMG MDA}

Just as expected, the total serum cholesterol of SIM-treated groups showed a decreasing tendency with time (Figure 2A). It is noted that serum cholesterol of the irradiated groups showed a slight but not significant increase compared to the respective control groups.

Electron-ray radiation induced a significant increase of MDA content in SMG in Groups III and IV when compared to Group I at all three time intervals (all $P<0.01$; Figure 2B). However, the extent of the increment in Group IV was less pronounced than that in Group III at 8 hours and 24 hours $(P<0.01)$ and returned to control levels by 4 weeks after IR.

\section{Histology}

SIM-alone treatment did not alter the histology of SMG when compared to the control group (Figure 3A and B). 
A

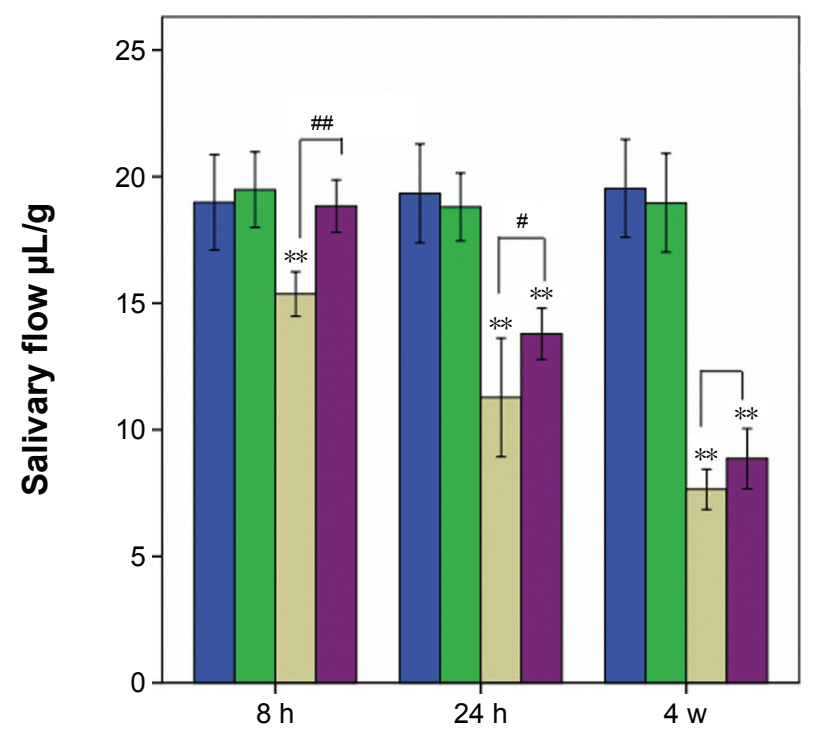

B

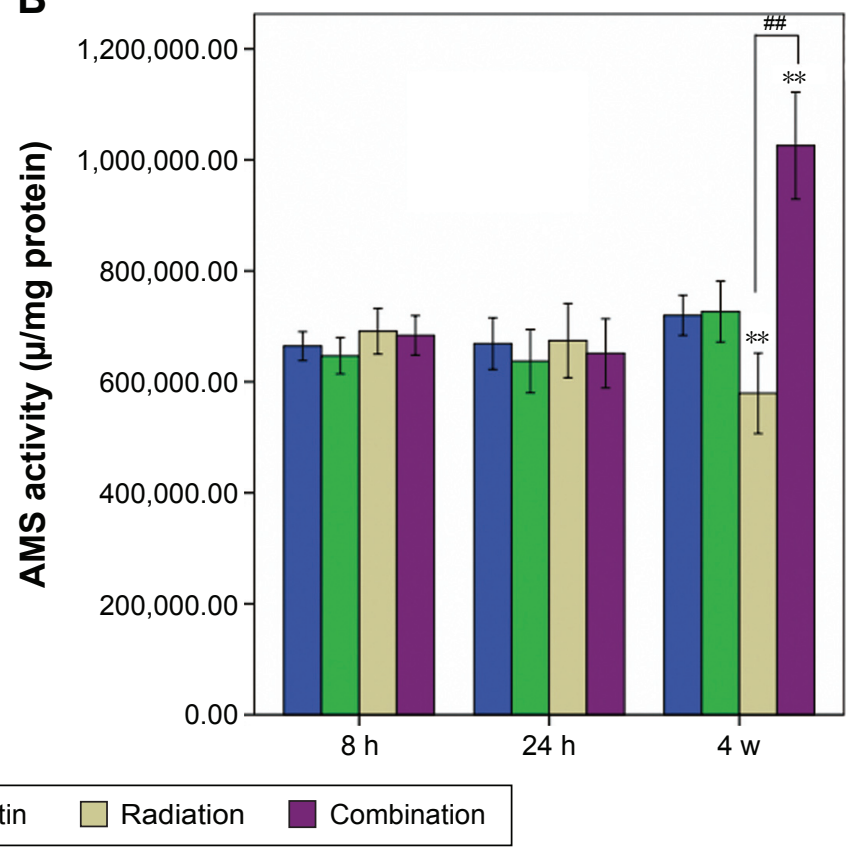

Figure I Salivary flow and AMS activity exam.

Notes: Saliva flow in a preclinical murine model of radiation-induced hyposalivation (A). Saliva was collected for 15 minutes after pilocarpine stimulation at 8 hours, 24 hours, and 4 weeks after 15 Gy irradiation and then normalized to body weight measured before collection $(n=8)$. Saliva flow of Group III (irradiation + solvent) presented with time-dependent decrease. While saliva flow of Group IV (irradiation + SIM) was not altered until 24 hours after IR, the extent of decrease was less than that of Group III at the other two time intervals. AMS activity of the four groups at different time intervals (B). AMS activity decreased only at 4 weeks after IR in IR-only group, while it increased significantly in the combination group at this time point. $* * P<0.0$ I, compared with the respective solvent group; ${ }^{\# P<0.05}$, \#P<0.0I, compared with Group IV.

Abbreviations: IR, irradiation; AMS, amylase; h, hours; w, weeks.

A

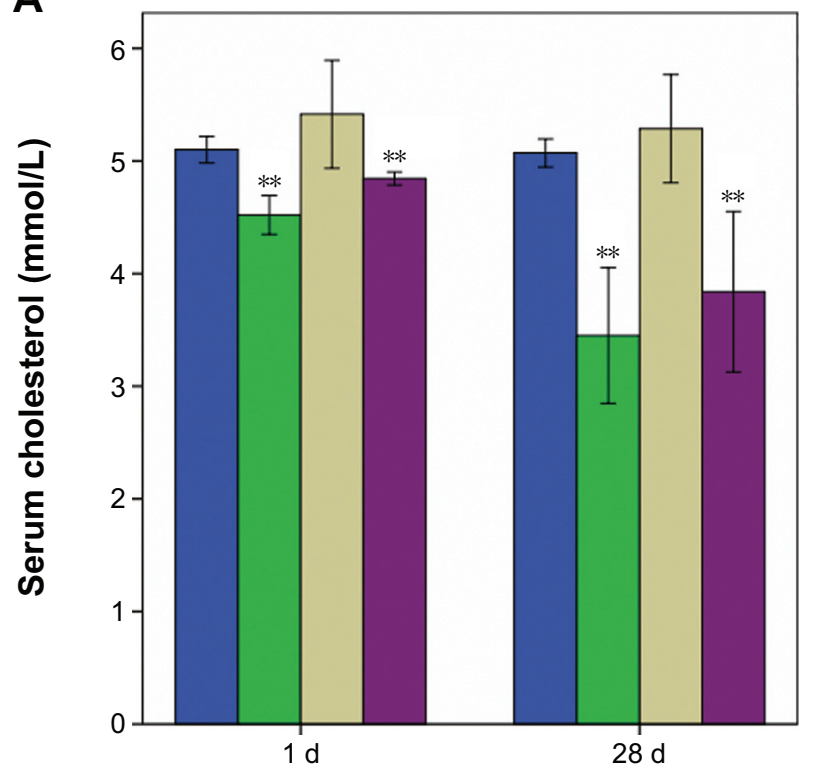

B

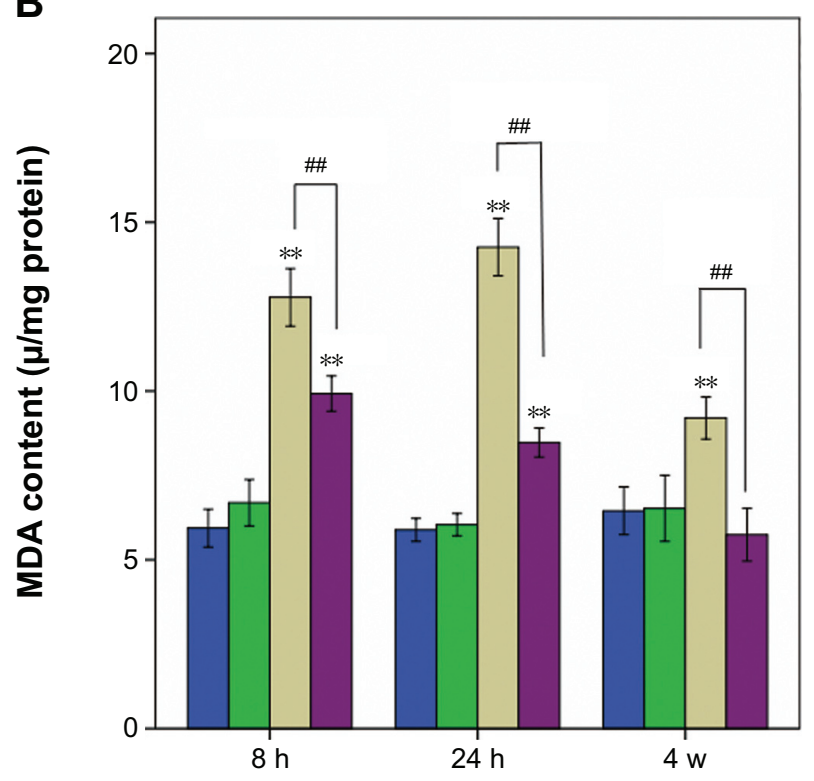

Control $\square$ Simvastatin $\square$ Radiation $\square$ Combination

Figure 2 Serum cholesterol and MDA content in SMGs of the four groups at different time intervals.

Notes: The total serum cholesterol of SIM-treated groups showed a decreasing tendency with time. Serum cholesterol of the irradiated groups showed a slight but not significant increase compared to the respective control groups (Figure 2A). Electron-ray radiation induced a significant increase of MDA content in SMG in Groups III (irradiation + solvent) and IV (irradiation + SIM) when compared to Group I (solvent + sham irradiation) at all three time intervals $(P<0.0 \mathrm{I}$, Figure $2 \mathrm{~B})$. The extent of the increment in Group IV was less pronounced than that in Group III at 8 hours and 24 hours and returned to control levels by 4 weeks after IR. Each group consisted of six samples. $* * P<0.0$ I, compared with the respective solvent group; ${ }^{\# P}<0.0$ I, compared with Group IV.

Abbreviations: MDA, malondialdehyde; SMG, submandibular gland; d, days; h, hours; w, weeks. 

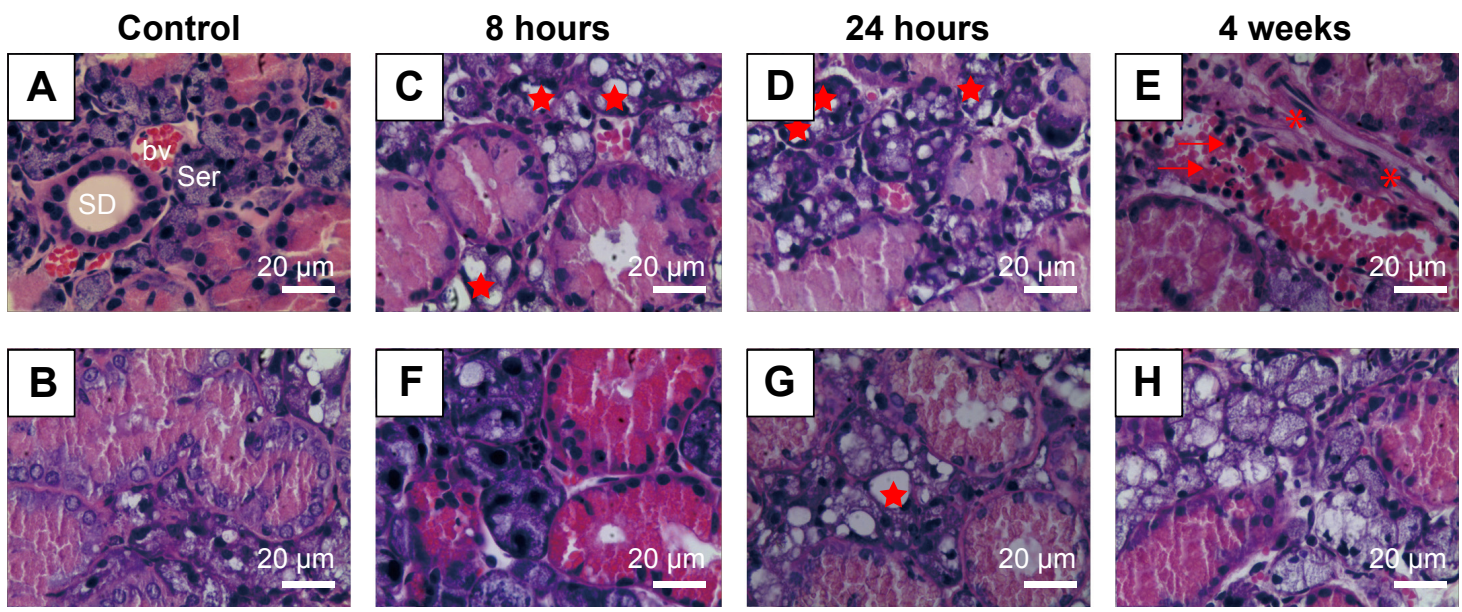

Figure 3 Histopathological changes in SMGs after irradiation (H\&E staining).

Notes: (A and B) Normal appearances of SMG in nonirradiated mice (A: Group I solvent + sham irradiation; B: Group II SIM + sham irradiation). (C-E) Histopathological changes in SMG of Group III (irradiation + solvent) at 8 hours, 24 hours, and 4 weeks separately after IR. (F-H) Histopathological changes in SMG of Group IV (irradiation + SIM) at the three time points. At 8 hours (C) and 24 hours (D), vacuolization of acinar cells and some pyknotic and polymorphic nuclei could be observed. At 4 weeks after IR (E), some inflammatory cells infiltration and focal fibrosis presented. Group IV showed clearer lobular structures, fewer vacuoles and inflammatory infiltration, and less fibration $(\mathbf{F}, \mathbf{G}, \mathbf{H})$. $\star$, vacuolization; $\rightarrow$, inflammatory cells infiltration; *, fibrosis. Original magnification, $\times 400$.

Abbreviations: SMG, submandibular gland; H\&E, hematoxylin and eosin; IR, irradiation; SD, striated duct; bv, blood vessel; Ser, seromucous acinus.

At 8 hours and 24 hours post-IR, the most obvious changes of Group III are vacuolization of acinar cells, but no clear pathological changes in the ducts or interstitium were observed (Figure 3C and D). In contrast, SIM-treated group showed clearer lobular structures, fewer vacuoles than that in Group III at 8 hours and 24 hours after IR (Figure $3 F$ and G). At 4 weeks, the most remarkable changes of interstitium in Group III are mononuclear infiltration and focal fibrosis (Figure 3E). However, no clear inflammatory infiltration or fibrosis was present at 4 weeks after IR, but some vacuoles (Figure 3H).

Peri-striated ductal and perivascular collagen deposition was demonstrated by Masson's trichrome stain (Figure 4). A very small amount of collagen deposition was seen in Group I (Figure 4A) and Group II (Figure 4B). A larger amount of collagen deposition could be observed in both Group III (Figure 4C) and Group IV (Figure 4D) at 4 weeks after IR compared with Group I. However, there was less collagen in Group III at 4 weeks after IR than that in Group IV.

\section{Effect of SIM on TGF- $\beta$ I expression by Western blot}

IR induced a striking increase of TGF- $\beta 1$ protein expression in Group III at 8 hours and 4 weeks after IR when compared with Group I (Figure 5). At 8 hours after IR, the amount of TGF- $\beta 1$ protein expression was increased $\sim 5.8$ fold in comparison to Group I. Moreover, a slight decrease in TGF- $\beta 1$ expression of Group III was observed at 24 hours after IR. This experiment was repeated six times using SMG from three mice, at this time point, and similar results were obtained. In contrast, we did not observe a significant increase in TGF- $\beta 1$ protein levels in Group IV at 8 hours, though we did see a modest increase at 24 hours, which then recovered to normal levels at 4 weeks.

\section{Discussion}

Our results suggest that SIM may serve as a novel alternative to radiation-induced hyposalivation, a severe complication of radiotherapy that impairs the life quality of hundreds of thousands of patients worldwide receiving radiotherapy for head and neck malignancies. Although the protective effects of statins in animal models of radiation injury have previously been investigated, ${ }^{9,11,12}$ our study is the first to demonstrate that SIM could protect salivary gland from radiation. Moreover, we report histological alteration of SMG after IR and the effects of SIM on MDA content and TGF- $\beta 1$ changes of SMG tissues.

It has been reported that hyperlipidemia developed after exposure to ionizing radiation, which results in the accumulation of cholesterol, triacylglycerols, and phospholipids. ${ }^{13,14}$ The results of our study showed slight but not significant elevation in serum cholesterol in the IR-treated mice. This could be attributed to regional IR rather than total body IR used, or different time intervals in other studies, which did not induce abundant release of cholesterol into peripheral blood. This 


\section{Control}
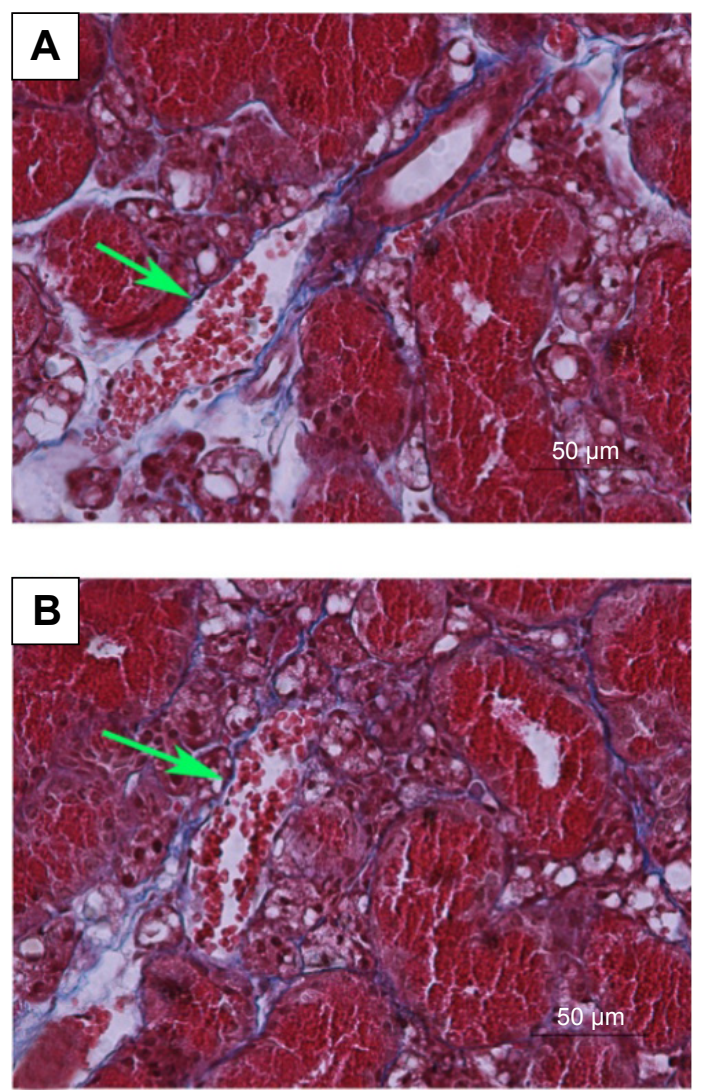

4 weeks after IR
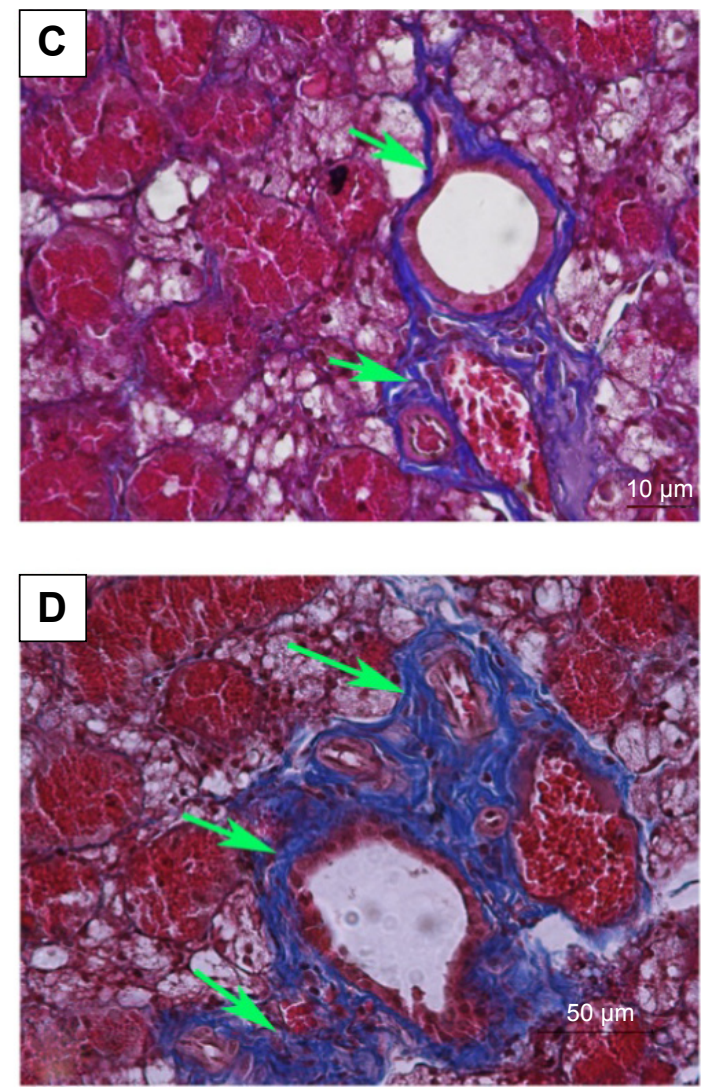

Figure 4 Masson's trichrome staining; collagen stained blue.

Notes: A small amount of collagen was seen in Group I (solvent + sham irradiation) (A) and Group II (SIM + sham irradiation) (B), while a large amount of collagen in Group III (irradiation + solvent) at 4 weeks after IR (C), but less than that in Group IV (irradiation + SIM) (D) could be observed (green arrows).

Abbreviation: IR, irradiation.

A

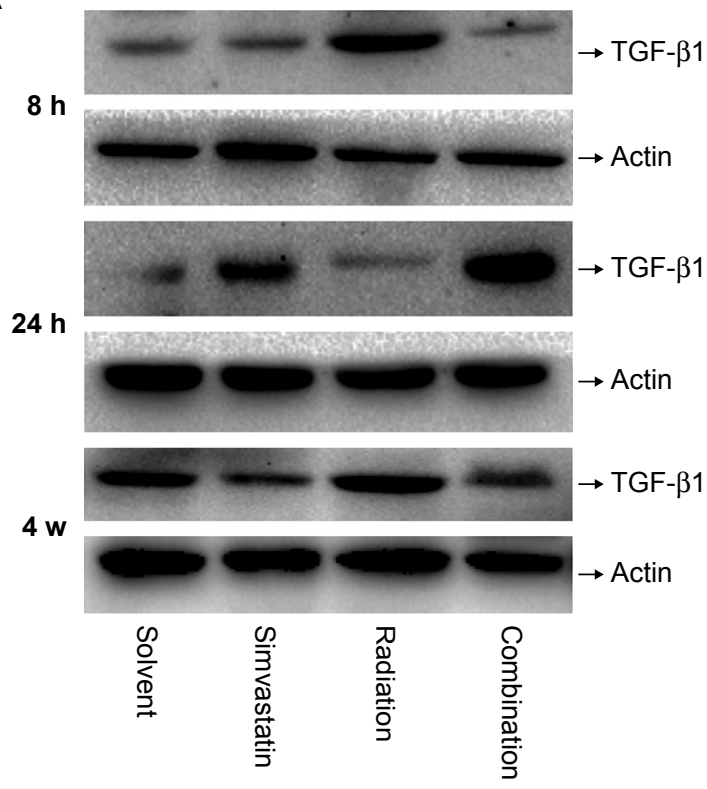

B

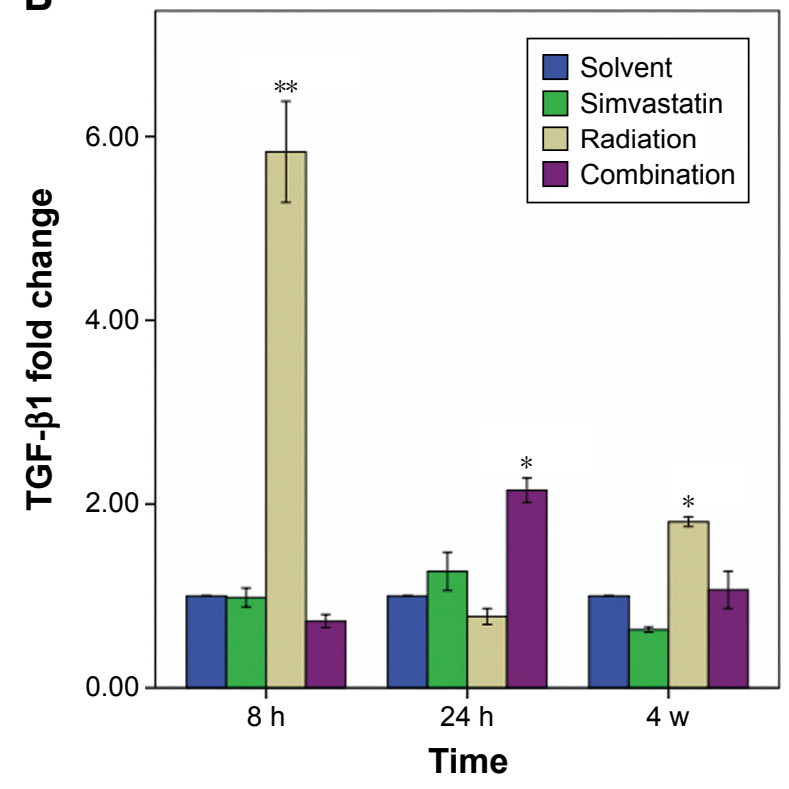

Figure 5 Protein content of TGF- $\beta$ I ( $25 \mathrm{Kd}$ ) in SMGs of mice at 8 hours, 24 hours, and 4 weeks after IR was assessed by Western blot analysis.

Notes: (A) Representative Western blots from three to five experiments with similar results. (B) Changes in TGF- $\beta$ I quantified by scanning densitometry analysis using Image Lab software. The data (relative density normalized to $\beta$-actin) are expressed as mean $\pm \mathrm{SD} ; * P<0.05, * * P<0.01$.

Abbreviations: TGF- $\beta$ I, transforming growth factor $\beta$ I; SMG, submandibular gland; IR, irradiation; SD, standard deviation; h, hours; w, weeks. 
test made sure that SIM exerted its lipid-lowering effect in the body of mice.

Exposure to ionizing radiation induces the production of reactive oxygen species, including superoxide, hydroxyl radicals, singlet oxygen, and hydrogen peroxide. ${ }^{15}$ Then, these free radicals damage proteins, lipids, and nucleic acids, resulting in cell dysfunction and death. ${ }^{16}$ Thus, the elimination of the free radical species from the cell environment could alleviate the side effects caused by IR.${ }^{17}$ In this study, SIM alone did not significantly alter the lipid peroxidation in unirradiated mice but SIM pretreatment significantly lowered the radiation-induced lipid peroxidation in terms of MDA production. Therefore, inhibition of lipid peroxidation by SIM is also of significance in protecting the cells from radiation-induced cell damage. So it may be proposed that the free radical scavenging ability of SIM may be one of the mechanisms for its radioprotective potential.

Following exposure to radiation, reactive oxygen species are produced, ${ }^{18}$ and these have been shown to be capable of activating latent TGF- $\beta 1 .{ }^{19}$ Large quantities of TGF- $\beta 1$ released from cells to the extracellular matrix could recruit inflammatory cells to an injury site, ${ }^{20}$ inhibition of the proliferation of epithelial cells, ${ }^{21}$ and enhancement of the maturation of fibroblasts into post-mitotic fibrocytes that increase production of fibrous tissue. ${ }^{22}$ Our histopathological findings further substantiated these observations. It was reported that TGF- $\beta 1$ increased significantly in mouse lung tissue after IR exposure, and suppression of TGF- $\beta 1$ resulted in a lower level of fibrosis in the lung of mice after IR. ${ }^{23,24}$ In this study, we found a pronounced overexpression of TGF- $\beta 1$ in response to IR after 8 hours and 4 weeks in normal SMG tissue. Administration of SIM could delay the rise and reduce the extent of elevation of TGF- $\beta 1$, which in turn protects the SMGs from radiation injury. Remarkably, a slightly lower expression of TGF- $\beta 1$ was observed compared with that of the normal group at 24 hours after IR, which presented nondecreasing tendency of TGF- $\beta 1$. To determine this issue, further studies are needed to detect the alteration of TGF- $\beta 1$ around 24 hours after IR.

\section{Conclusion}

Our findings demonstrated the potential of SIM as a radioprotective agent for SMG. These effects may be partly attributed to scavenging of radiation-induced free radicals and inhibiting collagen deposition and increases in TGF- $\beta 1$ expression. Based on the data presented here, we suggest that SIM may be clinically useful to alleviate side effects of radiotherapy on salivary gland, thus improving the overall therapeutic efficiency and tolerance of radiation-based tumor therapy.

\section{Acknowledgments}

This work was supported by the Natural Science Foundation of China (No 81272504), Research and Innovation Project for College Graduates of Jiangsu Province (No CXZZ12_0588), Innovation Team (No LJ201123 [EH11]), a project funded by the Priority Academic Program Development of Jiangsu Higher Education Institutions (PAPD) (JX10231801), and grants from Key Academic Discipline of Jiangsu Province "Medical Aspects of Specific Environments".

\section{Disclosure}

The authors report no conflicts of interest in this work.

\section{References}

1. Bansal M, Mohanti BK, Shah N, Chaudhry R, Bahadur S, Shukla NK Radiation related morbidities and their impact on quality of life in head and neck cancer patients receiving radical radiotherapy. Qual Life Res. 2004;13(2):481-488.

2. Nevens D, Nuyts S. The role of stem cells in the prevention and treatment of radiation-induced xerostomia in patients with head and neck cancer. Cancer Med. Epub 2016 Feb 16.

3. Pinna R, Campus G, Cumbo E, Mura I, Milia E. Xerostomia induced by radiotherapy: an overview of the physiopathology, clinical evidence, and management of the oral damage. Ther Clin Risk Manag. 2015; 11:171-188.

4. Liao JK, Laufs U. Pleiotropic effects of statins. Annu Rev Pharmacol Toxicol. 2005;45:89-118.

5. Greenwood J, Steinman L, Zamvil SS. Statin therapy and autoimmune disease: from protein prenylation to immunomodulation. Nat Rev Immunol. 2006;6(5):358-370.

6. Boz'entowicz-Wikarek M, Kocełak P, Smertka M, OlszaneckaGlinianowicz M, Chudek J. Effectiveness of lipid-lowering therapy with statins for secondary prevention of atherosclerosis - guidelines vs Reality. Pharmacol Rep. 2012;64(2):377-385.

7. Sobal G, Sinzinger H. Effect of simvastatin on the oxidation of native and modified lipoproteins. Biochem Pharmacol. 2005;70(8): $1185-1191$.

8. Wang J, Boerma M, Fu Q, Kulkarni A, Fink LM, Hauer-Jensen M. Simvastatin ameliorates radiation enteropathy development after localized, fractionated irradiation by a protein $\mathrm{C}$-independent mechanism. Int J Radiat Oncol Biol Phys. 2007;68(5):1483-1490.

9. Mathew B, Huang Y, Jacobson JR, et al. Simvastatin attenuates radiation-induced murine lung injury and dysregulated lung gene expression. Am J Respir Cell Mol Biol. 2011;44(3):415-422.

10. Ramos FM, Pontual ML, de Almeida SM, Boscolo FN, Tabchoury CP, Novaes PD. Evaluation of radioprotective effect of vitamin E in salivary dysfunction in irradiated rats. Arch Oral Biol. 2006;51(2):96-101.

11. Ostrau C, Hulsenbeck J, Herzog M, et al. Lovastatin attenuates ionizing radiation-induced normal tissue damage in vivo. Radiother Oncol. 2009; 92(3):492-499.

12. Williams JP, Hernady E, Johnston CJ, et al. Effect of administration of lovastatin on the development of late pulmonary effects after whole-lung irradiation in a murine model. Radiat Res. 2004;161(5):560-567.

13. Dubner D, Gisone P, Jaitovich I, Perez M. Free radicals production and estimation of oxidative stress related to gamma irradiation. Biol Trace Elem Res. 1995 Jan-Mar;47(1-3):265-270. 
14. Nada AS, Hawas AM, Amin Nel D, Elnashar MM, Abd Elmageed ZY. Radioprotective effect of Curcuma longa extract on gamma-irradiationinduced oxidative stress in rats. Can J Physiol Pharmacol. 2012;90(4):415-423.

15. Ewing D, Jones SR. Superoxide removal and radiation protection in bacteria. Arch Biochem Biophys. 1987;254(1):53-62.

16. Cerutti PA. Prooxidant states and tumor promotion. Science. 1985; 227(4685):375-381.

17. Hosseinimehr SJ, Azadbakht M, Mousavi SM, Mahmoudzadeh A, Akhlaghpoor S. Radioprotective effects of hawthorn fruit extract against gamma irradiation in mouse bone marrow cells. J Radiat Res. 2007;48(1):63-68.

18. Riley PA. Free radicals in biology: oxidative stress and the effects of ionizing radiation. Int J Radiat Biol. 1994;65(1):27-33.

19. Jobling MF, Mott JD, Finnegan MT, et al. Isoform-specific activation of latent transforming growth factor beta (LTGF-beta) by reactive oxygen species. Radiat Res. 2006;166(6):839-848.

20. Ashcroft GS. Bidirectional regulation of macrophage function by TGFbeta. Microbes Infect. 1999;1(15):1275-1282.
21. Boyd FT, Massague J. Transforming growth factor-beta inhibition of epithelial cell proliferation linked to the expression of a $53-\mathrm{kDa}$ membrane receptor. J Biol Chem. 1989;264(4):2272-2278.

22. Hakenjos L, Bamberg M, Rodemann HP. TGF-beta1-mediated alterations of rat lung fibroblast differentiation resulting in the radiationinduced fibrotic phenotype. Int J Radiat Biol. 2000;76(4):503-509.

23. Rube CE, Uthe D, Schmid KW, et al. Dose-dependent induction of transforming growth factor beta (TGF-beta) in the lung tissue of fibrosis-prone mice after thoracic irradiation. Int J Radiat Oncol Biol Phys. 2000;47(4):1033-1042.

24. Franko AJ, Sharplin J, Ghahary A, Barcellos-Hoff MH. Immunohistochemical localization of transforming growth factor beta and tumor necrosis factor alpha in the lungs of fibrosis-prone and "nonfibrosing" mice during the latent period and early phase after irradiation. Radiat Res. 1997;147(2):245-256.
Drug Design, Development and Therapy

\section{Publish your work in this journal}

Drug Design, Development and Therapy is an international, peerreviewed open-access journal that spans the spectrum of drug design and development through to clinical applications. Clinical outcomes, patient safety, and programs for the development and effective, safe, and sustained use of medicines are a feature of the journal, which

\section{Dovepress}

has also been accepted for indexing on PubMed Central. The manuscript management system is completely online and includes a very quick and fair peer-review system, which is all easy to use. Visit http://www.dovepress.com/testimonials.php to read real quotes from published authors.

Submit your manuscript here: http://www.dovepress.com/drug-design-development-and-therapy-journal 